\title{
Prognostic significance of electrical alternans versus signal averaged electrocardiography in predicting the outcome of electrophysiological testing and arrhythmia-free survival
}

\author{
A A Armoundas, D S Rosenbaum, J N Ruskin, H Garan, R J Cohen
}

\begin{abstract}
Objective-To investigate the accuracy of signal averaged electrocardiography (SAECG) and measurement of microvolt level $T$ wave alternans as predictors of susceptibility to ventricular arrhythmias. Design-Analysis of new data from a previously published prospective investigation.

Setting-Electrophysiology laboratory of a major referral hospital.

Patients and interventions-43 patients, not on class I or class III antiarrhythmic drug treatment, undergoing invasive electrophysiological testing had SAECG and $T$ wave alternans measurements. The SAECG was considered positive in the presence of one (SAECG-I) or two (SAECG-II) of three standard criteria. $T$ wave alternans was considered positive if the alternans ratio exceeded 3.0.

Main outcome measures-Inducibility of sustained ventricular tachycardia or fibrillation during electrophysiological testing, and 20 month arrhythmia-free survival.
\end{abstract}

Results-The accuracy of $T$ wave alternans in predicting the outcome of electrophysiological testing was $84 \%(p<0.0001)$. Neither SAECG-I (accuracy 60\%; p < 0.29 ) nor SAECG-II (accuracy $71 \%$; p $<0.10$ ) was a statistically significant predictor of electrophysiological testing. SAECG, $T$ wave alternans, electrophysiological testing, and follow up data were available in 36 patients while not on class $I$ or III antiarrhythmic agents. The accuracy of $T$ wave alternans in predicting the outcome of arrhythmia-free survival was $86 \%(p<0.030)$. Neither SAECG-I (accuracy $65 \%$; $p<0.21$ ) nor SAECG-II (accuracy $71 \%$; $p<0.48$ ) was a statistically significant predictor of arrhythmia-free survival.

Conclusions-T wave alternans was a highly significant predictor of the outcome of electrophysiological testing and arrhythmia-free survival, while SAECG was not a statistically significant predictor. Although these results need to be confirmed in prospective clinical studies, they suggest that $T$ wave alternans may serve as a non-invasive probe for screening high risk populations for malignant ventricular arrhythmias.

(Heart 1998;80:251-256)
Keywords: electrophysiological testing; electrical alternans; signal averaged electrocardiography; arrhythmias

The presence of late potentials in the signal averaged electrocardiogram (SAECG) has been used as a marker of susceptibility to ventricular tachyarrhythmias in a variety of patient populations. ${ }^{1-4}$ Late potentials are low amplitude signals in the ECG occurring in the terminal portion of the QRS complex thought to result from delayed or fractionated ventricular depolarisation. This delayed activity may play a role in initiating reentry and thus be a marker of susceptibility to reentrant ventricular tachyarrhythmias. Because the late potentials are so small in amplitude it is generally necessary to average over many beats in order to detect them. A clinical limitation of SAECG is that it cannot be used to evaluate patients with baseline prolongation of the unfiltered QRS complex owing to bundle branch block or other intraventricular conduction abnormalities.

Recently, the presence of microvolt level electrical alternans of the ST segment and the $T$ wave ( $T$ wave alternans) has been shown to be highly predictive of tachyarrhythmic events in patients undergoing electrophysiological testing. ${ }^{5}{ }^{6}$ In contrast to the SAECG, T wave alternans measures the beat to beat variability of the ECG wave about its mean value rather than the average ECG waveform. Also in contrast to SAECG, $T$ wave alternans characterises repolarisation processes rather than depolarisation processes. In addition, $T$ wave alternans - as opposed to SAECG - may be used to assess arrhythmic risk in patients with QRS prolongation as well as in patients with a normal QRS duration.

Our goal in the present study was to compare, in a retrospective analysis, $\mathrm{T}$ wave alternans with SAECG as predictors of the outcome of invasive electrophysiological testing and arrhythmia-free survival in the same patient population.

\section{Methods}

The prognostic accuracy of $\mathrm{T}$ wave alternans was compared with that of signal averaged electrocardiography with regard to the prediction of the outcome of electrophysiological testing and with arrhythmia-free survival. 
PATIENTS

We retrospectively analysed the medical records of patients from a previously published study $^{5}$ which examined the relation between $\mathrm{T}$ wave alternans, electrophysiological testing, and arrhythmia-free survival in patients scheduled to undergo electrophysiological testing. Since class I and III antiarrhythmic agents may affect $\mathrm{T}$ wave alternans and electrophysiological testing inducibility, ${ }^{7}$ and may also affect the SAECG independently of their effect on arrhythmia inducibility, ${ }^{89}$ we compared SAECG, T wave alternans, and electrophysiological testing in patients in whom all three measurements were made while they were not on antiarrhythmic drugs. The patients' characteristics are shown in table 1 . We identified 43 patients satisfying these criteria. Of these, 32 were male and 11 were female; their mean (SD) age was 56 (16) years.

In evaluating SAECG, $T$ wave alternans, and electrophysiological testing as predictors of arrhythmia-free survival we identified 36 patients (from among the 43 mentioned above) in whom the SAECG was measured while they were off class I and III antiarrhythmic agents and in whom follow up data were available in the absence of antiarrhythmic drug treatment. Of these, 24 were male and 12 were female; their mean (SD) age was 54 (17) years.

\section{ELECTROPHYSIOLOGICAL TESTING AND}

ARRHYTHMIA-FREE SURVIVAL

Electrophysiological testing was conducted under a standardised protocol in which up to three extrastimuli were delivered from two right ventricular sites. Electrophysiological testing outcome was classified as positive or negative according to previously established criteria. ${ }^{5}$ Arrhythmia-free survival was defined as survival in the absence of ventricular arrhythmic events (documented sustained ventricular tachycardia, ventricular fibrillation, or sudden cardiac death). ${ }^{5}$

Table 1 Characteristics of the 43 patients

\begin{tabular}{lc}
\hline & Number of patients \\
\hline Type of heart disease & $23(53 \%)$ \\
Coronary artery disease & $4(9 \%)$ \\
Congestive cardiomyopathy & $6(14 \%)$ \\
Wolff-Parkinson-White syndrome & $1(2 \%)$ \\
Other & $9(21 \%)$ \\
No organic heart disease & $12(28 \%)$ \\
Ejection fraction < 40\% & \\
& $18(42 \%)$ \\
History of myocardial infarction & \\
Indications for electrophysiological study & $3(7 \%)$ \\
Symptomatic ventricular activity & $9(21 \%)$ \\
Sustained VT & $9(21 \%)$ \\
Ventricular fibrillation & $10(23 \%)$ \\
Syncope & $11(26 \%)$ \\
Supraventricular tachycardia & $1(2 \%)$ \\
Other & \\
Arrhythmia induced & $23(43 \%)$ \\
Negative & $4(9 \%)$ \\
Non-sustained VT & $4(9 \%)$ \\
Sustained monomorphic VT & $6(14 \%)$ \\
Ventricular fibrillation & $6(14 \%)$ \\
Supraventricular tachycardia &
\end{tabular}

Two patients had no ejection fraction data. Percentages do not total $100 \%$ because of rounding.

VT, ventricular tachycardia.

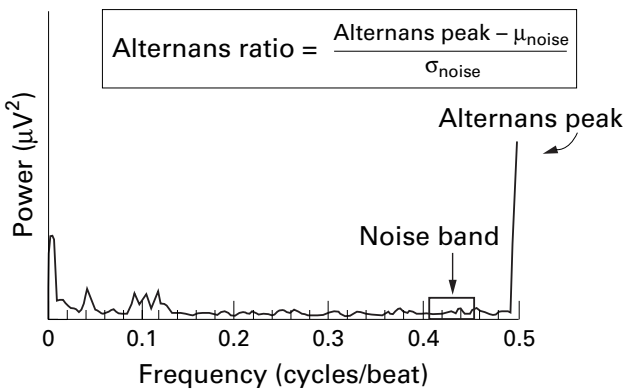

Figure 1 Representative example of power spectrum of beat to beat fluctuations in $T$ wave morphology. The alternans ratio is the amplitude of the spectrum at the alternans frequency (alternans peak) minus the mean background noise level (noise), divided by the standard deviation of the noise (noise) in the reference noise band.

T WAVE ALTERNANS TESTING

Electrical alternans of the $\mathrm{T}$ wave was computed, as previously reported, ${ }^{5}$ by analysis of the vector magnitude ECG signal derived from the three Frank orthogonal leads. The vector magnitude signal was analysed using a spectral analysis algorithm which can detect microvolt level alternans. ${ }^{10}$ Electrical alternans represents subtle (microvolt level) changes in the morphology of electrocardiographic complexes occurring on an every other beat basis. The spectral method of detecting such low level changes involves analysis of 128 consecutive ECG complexes. Fourier analysis methods are used to compute the power spectra of the beat to beat fluctuations in the amplitudes of corresponding sample points of the 128 time aligned complexes. The power spectra corresponding to sample points within a given section of ECG complex (for example, $\mathrm{T}$ wave) are averaged. The presence of alternans is indicated by the presence of a peak at the last point in the averaged spectrum, corresponding to a frequency of 0.5 cycles per beat (fig 1 ). The number of standard deviations of the baseline noise by which the last point in the power spectrum exceeds the mean level of the noise in the adjacent reference noise band is called the alternans ratio $(\mathrm{k})$. The alternans ratio is a measure of the statistical significance of the alternans. Patients were classified positive for the presence of electrical alternans if the alternans ratio of the $T$ wave exceeded 3.0. ${ }^{5}$

SAECG TESTING

SAECG data that had been recorded when the patients were in the hospital were analysed. For the SAECG test either an MAC 15 (Marquette Electronics, Milwaukee, Wisconsin, USA) or a Predictor (Corazonix Inc, Oklahoma, Oklahoma, USA) were used for data collection. In both systems the averaged QRS complexes were filtered with a spectral bandpass filter with a high pass frequency of $40 \mathrm{~Hz}$ and a low pass frequency of $250 \mathrm{~Hz}$. In the MAC 15 system the spectral filter was implemented by means of a fast Fourier transform, while in the Predictor the spectral filter was a four pole bidirectional Butterworth filter.

The criteria for abnormality of the three signal averaged electrocardiographic variables for the Predictor were: (1) filtered QRS duration 
Table 2 Twave alternans (TWA), signal averaged electrocardiography (SAECG, one or two criteria), and combination of TWA and SAECG as predictors of inducible ventricular arrhythmias at electrophysiological testing

\begin{tabular}{lllllllll}
\hline & $n$ & Sensitivity & Specificity & $P V+$ & $P V-$ & $R R$ & Accuracy & p value \\
\hline TWA & 43 & $70 \%$ & $88 \%$ & $64 \%$ & $90 \%$ & 6.8 & $84 \%$ & $<0.0001$ \\
SAECG-I & 35 & $50 \%$ & $62 \%$ & $21 \%$ & $86 \%$ & 1.5 & $60 \%$ & $<0.29$ \\
SAECG-II & 35 & $50 \%$ & $76 \%$ & $30 \%$ & $88 \%$ & 2.5 & $71 \%$ & $<0.10$ \\
TWA+SAECG-I & 43 & $60 \%$ & $94 \%$ & $75 \%$ & $89 \%$ & 6.6 & $86 \%$ & $<0.0001$ \\
TWA+SAECG-II & 43 & $60 \%$ & $97 \%$ & $86 \%$ & $80 \%$ & 7.7 & $88 \%$ & $<0.0001$
\end{tabular}

$\overline{\mathrm{n} \text {, number of cases with a determinate test result; } \mathrm{PV}+\text {, positive predictive value; } \mathrm{PV}-\text {, negative }}$ predictive value; $R R$, relative risk.

(QRSD) > $114 \mathrm{~ms}$; (2) low amplitude signal duration (LAS) > $38 \mathrm{~ms}$; and (3) root mean square voltage of the last $40 \mathrm{~ms}$ (RMSV) $<20 \mu \mathrm{V}$. $^{11}$

Since the MAC 15 uses an FFT filter we applied the linear regression analysis of Cristenson $e t a l^{12}$ to calculate a set of thresholds for the signal averaged electrocardiographic variables, measured with the MAC 15 equivalent to the thresholds used with the Predictor. We used the following equations: (1) QRS duration, $\mathrm{y}=0.97 \mathrm{x}+13.8$, and $\mathrm{R}^{2}=0.99 ;$ (2) $L A S$, $\mathrm{y}=1.06 \mathrm{x}-1.19$, and $\mathrm{R}^{2}=0.92$; (3) $R M S V$, $\mathrm{y}=0.88 \mathrm{x}-0.97$ and $\mathrm{R}^{2}=0.88$, where $\mathrm{y}$ represents thresholds for measurements made with the FFT filter, $\mathrm{x}$ represents thresholds for measurements made with the bidirectional filter, and $\mathrm{R}^{2}$ is the statistic used to assess the quality of the regression model. These equations provided the following threshold values for the Marquette system: (1) filtered QRS duration (QRSD) > $124 \mathrm{~ms}$; (2) low amplitude signal duration (LAS) $>39 \mathrm{~ms}$; and (3) root mean square voltage of the last $40 \mathrm{~ms}$ (RMSV) $<17 \mu \mathrm{V}$

Two commonly used sets of criteria were employed to classify the outcome of the SAECG test: SAECG-I was positive if one or more SAECG variables were abnormal and negative otherwise, SAECG-II if two or more SAECG variables were abnormal and negative otherwise. A patient was deemed indeterminate for SAECG if the unfiltered QRS duration was greater than $120 \mathrm{~ms}$; the duration of the unfiltered QRS was defined as the longest QRS duration in any of the Frank orthogonal leads $(\mathrm{X}, \mathrm{Y}, \mathrm{Z})$.

COMBINED T WAVE ALTERNANS AND SAECG MEASURES

In addition to reporting the $\mathrm{T}$ wave alternans and SAECG results we also retrospectively developed combined measures ( $\mathrm{T}$ wave alternans + SAECG-I, T wave alternans + SAECGII); a patient was classified positive for these measures if $\mathrm{T}$ wave alternans was positive and the SAECG (SAECG-I or SAECG-II respectively) was positive or indeterminate, and negative otherwise.

\section{STATISTICS}

We used $\chi^{2}$ analysis to analyse the relation of measures of SAECG and T wave alternans with electrophysiological testing inducibility. The relation between these measures and arrhythmia-free survival was analysed using Kaplan-Meier survival analysis and the Cox proportional hazard model; $p$ values for Kaplan-Meier survival analysis were computed using the log-rank test. All $\mathrm{p}$ values reflect single sided tests; $p$ values less than 0.05 were considered significant.

\section{Results}

PREDICTION OF ELECTROPHYSIOLOGICAL TESTING INDUCIBILITY

We compared the accuracy of $\mathrm{T}$ wave alternans, SAECG, $T$ wave alternans + SAECG-I, and $\mathrm{T}$ wave alternans + SAECG-II in predicting the outcome of electrophysiological testing in 43 patients (table 2 ) using $\chi^{2}$ analysis. In this study of $\mathrm{T}$ wave alternans, conducted during atrial pacing, all patients who met the entry criteria (as reported in Rosenbaum et $a \bar{P}$ ) had determinate $\mathrm{T}$ wave alternans tests. Eight patients were classified as indeterminate for the SAECG test owing to prolonged QRS duration. In the remaining 35 patients neither SAECG-I nor SAECG-II were statistically significant predictors of inducibility at electrophysiological testing. Four of the patients indeterminate for SAECG were inducible at electrophysiological testing. In comparison, $\mathrm{T}$ wave alternans predicted electrophysiological testing inducibility with a sensitivity of $70 \%$, a specificity of $88 \%$, and a relative risk of 6.8 $(\mathrm{p}<0.0001)$. Both $\mathrm{T}$ wave alternans + SAECG-I and T wave alternans + SAECG-II were statistically significant predictors of electrophysiological testing.

We also evaluated the accuracy of $\mathrm{T}$ wave alternans in predicting the outcome of electrophysiological testing in the group of patients who were not indeterminate for SAECG. We found that $T$ wave alternans predicted electrophysiological testing inducibility with a sensitivity of $67 \%$, a specificity of $90 \%$, a positive predictive value of $57 \%$, a negative predictive value of $93 \%$, and a relative risk of 8.0 $(\mathrm{p}<0.00085)$.

Multivariate stepwise logistic regression analysis including SAECG results, to identify independent predictors of inducible ventricular tachycardia, did not provide a better model than that presented by Rosenbaum et al. ${ }^{13}$ This model included ejection fraction or history of myocardial infarction as one predictor variable, and $\mathrm{T}$ wave alternans as the second.

PREDICTION OF ARRHYTHMIA-FREE SURVIVAL Of 36 patients who were followed up (range 2.9 to 19.9 months, median 5.7 months), three (8\%) developed spontaneous ventricular tachycardia or ventricular fibrillation. The median time to arrhythmia occurrence was 4.6 months (range 3.8 to 12 months) from the time of the electrophysiological testing study. Five of the 36 patients had indeterminate SAECG tests owing to QRS prolongation.

Kaplan-Meier actuarial survival analysis was used to ascertain the ability of electrophysiological testing, $\mathrm{T}$ wave alternans, and SAECG to predict long term arrhythmia-free survival (figs 2-4). Table 3 compares the accuracy of electrophysiological testing, $\mathrm{T}$ wave alternans, SAECG-I, SAECG-II, T wave alternans + SAECG-I, and T wave alternans + SAECG-II testing in predicting 20 month actuarial arrhythmia-free survival. Here 


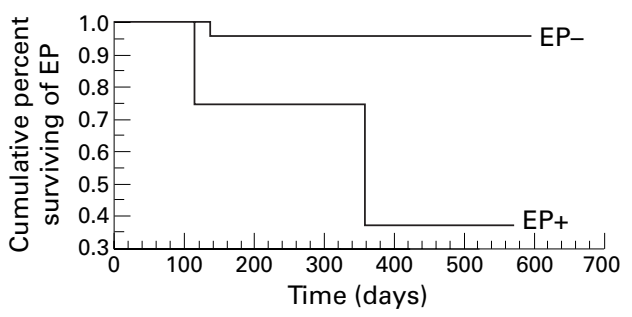

Figure 2 Arrhythmia-free survival of patients with positive/negative electrophysiological testing (EP).

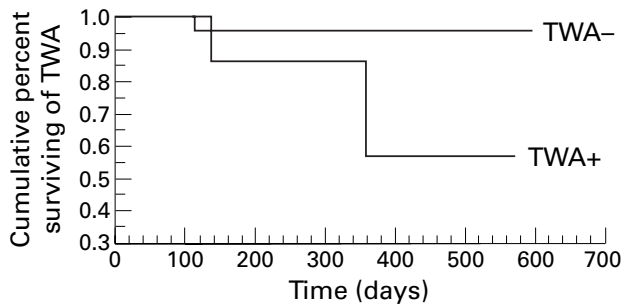

Figure 3 Arrhythmia-free survival of patients with positive/negative $T$ wave alternans (TWA).

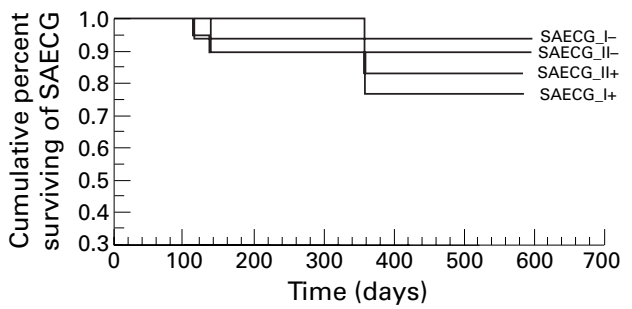

Figure 4 Arrhythmia-free survival of patients with positive/negative signal averaged electrocardiography (SAECG, one or two criteria).

electrophysiological testing and $\mathrm{T}$ wave alternans were statistically significant predictors of arrhythmia-free survival, while SAECG-I and SAECG-II were not. None of the indeterminate patients for SAECG had an arrhythmic event during follow up. The combined measure T wave alternans + SAECG-I was also a statistically significant predictor of arrhythmia-free survival.

We also evaluated the accuracy of electrophysiological testing and $\mathrm{T}$ wave alternans in predicting arrhythmia-free survival in the group of patients that were not indeterminate for SAECG. We found that electrophysiological testing had a sensitivity of $67 \%$, a specificity $96 \%$, a positive predictive value $67 \%$, a negative predictive value of $96 \%$, and a relative risk of 18.7 ( $\mathrm{p}<0.0005$ ), while $\mathrm{T}$ wave alternans had a sensitivity of $60 \%$, a specificity $89 \%$, a positive predictive value $40 \%$, a negative predictive value of $96 \%$, and a relative risk of 10.4 $(\mathrm{p}<0.013)$.

Table 3 Electrophysiological (EP) testing, T wave alternans (TWA), signal averaged electrocardiography (SAECG, one or two criteria), and combination of TWA and SAECG as predictors of actuarial 20 month arrhythmia-free survival

\begin{tabular}{lllllllll}
\hline & $n$ & Sensitivity & Specificity & PV+ & $P V-$ & $R R$ & Accuracy & p value \\
\hline EP testing & 36 & $67 \%$ & $94 \%$ & $50 \%$ & $97 \%$ & 16.0 & $92 \%$ & $<0.0012$ \\
TWA & 36 & $75 \%$ & $88 \%$ & $43 \%$ & $97 \%$ & 12.4 & $86 \%$ & $<0.030$ \\
SAECG-I & 31 & $75 \%$ & $63 \%$ & $30 \%$ & $94 \%$ & 5.4 & $65 \%$ & $<0.21$ \\
SAECG-II & 31 & $50 \%$ & $74 \%$ & $22 \%$ & $91 \%$ & 2.4 & $71 \%$ & $<0.48$ \\
TWA+SAECG-I & 36 & $75 \%$ & $94 \%$ & $60 \%$ & $97 \%$ & 18.6 & $92 \%$ & $<0.0047$ \\
TWA+SAECG-II 36 & $50 \%$ & $94 \%$ & $50 \%$ & $94 \%$ & 8.0 & $89 \%$ & $<0.13$
\end{tabular}

$\mathrm{n}$, number of cases with a determinate test result; $\mathrm{PV}+$, positive predictive value; $\mathrm{PV}-$, negative predictive value; $R R$, relative risk.
Using a Cox proportional hazard model, electrophysiological testing $\quad\left(\chi^{2}=4.95\right.$, $\mathrm{p}<0.013), \mathrm{T}$ wave alternans $\left(\chi^{2}=2.77\right.$, $\mathrm{p}<0.048)$, and T wave alternans + SAECG-I $\left(\chi^{2}=4.048, \mathrm{p}<0.022\right)$ were found to be significant univariate predictors of 20 month arrhythmia-free survival, while SAECG-I $\left(\chi^{2}=0.61, \mathrm{p}<0.22\right)$, SAECG-II $\left(\chi^{2}=0.0033\right.$, $\mathrm{p}<0.48)$, and T wave alternans + SAECG-II $\left(\chi^{2}=0.81, \mathrm{p}<0.18\right)$ were not.

T WAVE ALTERNANS AND SAECG POSITIVITY IN PATIENTS WITH HISTORY OF VENTRICULAR TACHYCARDIA OR VENTRICULAR FIBRILLATION The incidence of $T$ wave alternans positivity in the group of 18 patients with a history of ventricular tachycardia or ventricular fibrillation was $44 \% v 12 \%$ in the group of 25 patients with no such history. For SAECG-I (SAECGII), of the 13 patients with a history of ventricular tachycardia or ventricular fibrillation with determinate SAECG tests 38\% $(23 \%)$ were positive, and of the 22 patients without such a history but with determinate SAECG tests, $41 \%$ (32\%) were positive.

\section{Discussion}

Sudden cardiac death remains an important public health problem. The availability of effective treatment in terms of the implantable cardioverter/defibrillator makes it critical to be able to identify individuals at risk. An essential step in this process is the development of noninvasive techniques to screen patients who may be at risk. To date the detection of delayed ventricular activation using the signal averaged electrocardiogram has been a non-invasive technique used for this purpose. Although SAECG has also been shown to predict susceptibility to inducible ventricular arrhythmias, it has a low positive predictive value. In addition time domain signal averaging is applicable to a limited patient population since late potentials are not detectable in the presence of intraventricular conduction abnormalities such as bundle branch block. Moreover, the SAECG evolves in time following myocardial infarction. ${ }^{14} 15$

We compared SAECG with electrical alternans a new potentially non-invasive method of assessing susceptibility to ventricular arrhythmias. In this study conducted retrospectively in patients referred for electrophysiological testing, we found that $T$ wave alternans was an accurate predictor of the outcome of invasive electrophysiological testing and arrhythmiafree survival, while SAECG alone was not. Furthermore, many of the SAECG tests were indeterminate because of prolonged unfiltered QRS duration. Intraventricular conduction blocks did not prevent the detection of electrical alternans.

Our results are consistent with those of Estes et al who also found that SAECG was not a statistically significant predictor of inducibility at electrophysiological testing in a population of 27 patients in which $\mathrm{T}$ wave alternans was statistically significant. ${ }^{16}$ Turitto et al found in 105 patients with non-sustained ventricular tachycardia on Holter monitoring that SAECG 
was a predictor of inducible ventricular tachycardia, with a sensitivity in the $64 \%$ to $73 \%$ range and a specificity in the $71 \%$ to $89 \%$ range, ${ }^{17}$ similar to the values of sensitivity $(50 \%)$ and specificity $(62 \%$ to $76 \%)$ for SAECG obtained here. In a large study of 2461 postmyocardial infarction patients, Savard et al found that SAECG was a statistically significant predictor of arrhythmic events during a one to two year follow up, ${ }^{18}$ with a sensitivity of $65 \%$ and a specificity of $68.4 \%$, similar to the values of sensitivity ( $50 \%$ to $75 \%$ ) and specificity $(63 \%$ to $74 \%)$ found here. Another study in postmyocardial infarction patients with complex ventricular arrhythmias reported no significant relation between SAECG and the occurrence of sudden death. ${ }^{19}$ Thus the values of sensitivity and specificity for SAECG as a predictor of electrophysiological testing or future arrhythmic events obtained here are similar to values obtained in other studies. In some of the larger studies, SAECG achieves statistical significance with moderate values of sensitivity and specificity. Of course, it is not possible to compare directly values of sensitivity and specificity across different studies because these values may be highly dependent on the particular patient population. In the population we studied, $\mathrm{T}$ wave alternans provided a more sensitive and specific measure of arrhythmia susceptibility than SAECG.

The accuracy of $T$ wave alternans was slightly improved, retrospectively, in a combined test ( $T$ wave alternans + SAECG-I) using both SAECG and T wave alternans data. In this combined test a positive outcome required a positive $T$ wave alternans test and a non-negative SAECG result, thus eliminating occasional false positives in $\mathrm{T}$ wave alternans testing. It seems reasonable that $\mathrm{T}$ wave alternans, which is a measure of repolarisation, may contain different information with respect to ventricular susceptibility to arrhythmias than SAECG, which is a measure of depolarisation. Further, one might expect non-overlapping information in $\mathrm{T}$ wave alternans and SAECG because $T$ wave alternans is a measure of beat to beat variability, whereas SAECG is a measure of mean QRS morphology.

In this study delayed conduction alone, as measured in the SAECG, did not identify a myocardial substrate at risk for ventricular arrhythmias. In contrast, repolarisation alternans did appear to identify a substrate susceptible to reentrant arrhythmia. The development of alternans may indicate the presence of a dispersion in refractoriness resulting in some regions of the myocardium not being normally activated on alternate beats, thus leading to alternating patterns of conduction detected as microvolt level alternans in the surface ECG. These same regions of myocardium may constitute islands of fully or partially refractory tissue on alternate beats, promoting slowed conduction, wavefront fractionation, and reentry. ${ }^{20}{ }^{21}$ Thus the underlying dispersion of recovery may both generate the alternans and promote the development of reentrant arrhythmias. Alternatively, microvolt level alternans in the surface ECG may relate to action potential alternans in individual myocardial cells; in this case the alternans itself may be the source of the increased dispersion of recovery leading to wavefront fractionation and reentry.

\section{STUDY LIMITATIONS}

This study shows that $T$ wave alternans alone or in combination with SAECG may be an accurate indicator of arrhythmia susceptibility in a population of patients referred for electrophysiological testing. The current study is limited in two respects. First, electrical alternans was measured in patients in the electrophysiology laboratory during atrial pacing. Atrial pacing was used to increase the heart rate and eliminate beat to beat variability in heart rate which can interfere with the measurement of alternans. It has recently been shown that alternans measured without pacing during exercise has similar predictive accuracy to alternans measured during pacing. ${ }^{16}$ However, future studies will need to compare $\mathrm{T}$ wave alternans measured without the use of pacing with other predictors of ventricular arrhythmias. ${ }^{22}$ Second, the population selected for electrophysiological testing was used in this study because of the high incidence of arrhythmias in this group. However, the practical use of $\mathrm{T}$ wave alternans as a screening technique must be demonstrated in a lower risk population more similar to that encountered in clinical practice where screening would be applied. For example, a population of postmyocardial infarction patients or patients with heart failure would be an appropriate group to study. Given the lower expected event rate, such a study would have to be much larger than the one reported here to obtain statistically significant results.

\section{CONCLUSIONS}

In summary $\mathrm{T}$ wave alternans appears to be a useful new technique for identifying individuals at risk for ventricular arrhythmias. In this study $\mathrm{T}$ wave alternans compared favourably with SAECG. It is conceptually attractive to consider combining these two measures because SAECG measures depolarisation processes while $\mathrm{T}$ wave alternans is a measure of repolarisation processes. Furthermore, SAECG is a measure of beat averaged conduction whereas $\mathrm{T}$ wave alternans is a measure of beat to beat variability. Thus one might expect that these two measures would provide independent information on the risk of ventricular arrhythmias. However, in this study retrospectively combining $\mathrm{T}$ wave alternans with SAECG data was only slightly better than the $\mathrm{T}$ wave alternans results alone. In addition, $\mathrm{T}$ wave alternans was successfully measured in a larger proportion of patients than SAECG, because an intraventricular conduction delay does not interfere with determination of the former while it makes the latter uninterpretable. It will be important in future studies to compare $\mathrm{T}$ wave alternans with other noninvasive measures of arrhythmic vulnerability such as heart rate variability, ambient levels of ventricular ectopy, baroreceptor sensitivity, and QT interval dispersion. 
Supported by a grant from the National Space Biomedical Research Institute, NASA grant Nos NAGW-3297 and NAG54989 (to RJC), NIH grant No HL-54807, and a Whitake Foundation grant (to DSR). We thank Hartley Holden for hi technical assistance.

1 Gomez JA, Winters SL, Stewart D, et al. A new noninvasive index to predict sustained ventricular tachycardia and sudden death in the first year after myocardial infarction: based
on signal-averaged electrocardiogram, radionuclide ejecon signal-averaged electrocardiogram, radionuclide ejec-
tion fraction and Holter monitoring. $\mathcal{F} \mathrm{Am}$ Coll Cardio tion fraction and

2 Simson MB. Signal averaging. Circulation 1987;75(suppl III): $69-73$

3 Gomez JA, Winters SL, Martinson M, et al. The prognostic significance of quantitative signal-averaged variables relative to clinical variables, site of myocardial infarction, ejection fraction and ventricular premature beats: a prospective study. F Am Coll Cardiol 1989;13:377-84.

4 Kuchar DL, Thorburn CW, Sammel NL. Prediction of serious arrhythmic events after myocardial infarction: signaloused electrocardiogram, Holter monitoring and radionuclide ventriculography. $\mathcal{F}$ Am Coll Cardiol 1987;9:531-8.

5 Rosenbaum DS, Jackson LE, Smith JM, et al. Electrical Rosenbaum DS, Jackson LE, Smith JM, et al. Electrical alternans and vulnerability to

6 Armoundas AA, Cohen RJ. Clinical utility of T wave alternans. Cardiac Electrophysiol Rev 1997;1:390-4.

7 Zipes DP. Management of cardiac arrhythmias: pharmacological, electrical and surgical techniques. In: Braunwald E, ed. Heart disease, 4 th ed. Philadelphia: WB Saunders, 1992:628-66.

8 Steinber JS, Freedman RA, Bigger JT, the ESVEM Investigators. Antiarrhythmic drug therapy and the signalaveraged electrocardiogram. In: EL-Sherif N, Turitto G, eds. High resolution electrocardiography. Mount Kisco, NY: Futura Publishing Co, 1992:569-90

9 Goedel-Meinen L, Hofmann G, Schmidt G, et al. Amiodarone-efficacy and late potentials during long-term therapy. Int $\mathcal{F}$ Clin Pharmacol Ther Toxicol 1990;28:449-54.

10 Smith JM, Clancy EA, Valeri R, et al. Electrical alternans and cardiac electrical instability. Circulation 1988;77:110-21.

11 Breithardt GM, Cain E, El-Sherif N, et al. Standards for analysis of ventricular late potentials using high resolution or signal-averaged electrocardiography. Eur Heart J 1991; 12:473-80.
12 Christenson DW, Reddy BRS, Rowlandson GI. Filtering techniques for detection of late potentials. Proceedings of the First International High-Resolution ECG Seminar, Stockholm, 21-22 September 1990.

13 Rosenbaum DS, He B, Cohen RJ. New approaches for evaluating cardiac electrical activity: repolarization alternans and body surface Laplacian imaging. In: Zipes DP, Jalife J, eds. Cardiac electrophysiology: from cell to bedside, 2nd ed. Philadelphia: WB Saunders, 1995:1187-97.

14 Turitto G, Caref E, Macina G, et al. Time course of ventricular arrhythmias and the signal averaged electrocardiogram in the postinfarction period: a prospective study of correlation. Br Heart f 1988;60:17-22.

15 El-Sherif N, Ursell SN, Bekheit S, et al. Prognostic significance of the signal-averaged ECG depends on the time of recording in the postinfarction period. Am Heart $\mathcal{F}$ 1989;118:256-64.

16 Estes NAM, Michaud G, Zipes DP, et al. Electrical alternans during rest and exercise as predictors of vulnerability to ventricular arrhythmias. Am $\mathcal{f}$ Cardiol 1997;80:1314-18.

17 Turitto G, Fontaine JM, Ursell SN, et al. Value of signal averaged electrocardiogram as a predictor of the results of programmed stimulation in nonsustained ventricular tachycardia. Am f Cardiol 1988;61:1272-8.

18 Savard P, Rouleau JL, Ferguson J, et al. Risk stratification after myocardial infarction using signal-averaged electrocardiographic criteria adjusted for sex, age, and myocardial infarction location. Circulation 1997;96:202-13.

19 Mercando AD, Aronow WS, Epstein S, et al. Signalaveraged electrocardiography and ventricular tachycardia as predictors of mortality after acute myocardial infarction in elderly patients. Am f Cardiol 1995;76:436-40.

20 Smith JM, Cohen RJ. Simple finite-element model accounts for wide range of cardiac dysrhythmias. Proc Nat Acad Sci Med Sci 1984;81:233-7.

21 Clancy EA, Smith JM, Cohen RJ. A simple electricalmechanical model of the heart applied to the study of electrical-mechanical alternans. IEEE Trans Biomed Eng 1991;38:551-60.

22 Platt SB, Vijgen JM, Albrecht P, et al. Occult T wave alternans in long QT syndrome. F Cardiovasc Electrophysiol 1996; $7: 144-8$. 